\title{
ASCOMYCETES Y BASIDIOMYCETES MACROSCÓPICOS EN BOSQUES DE PUERTO ALMENDRAS (LORETO, PERÚ)
}

Teresa MORI DEL ÁGUILA', María E. BENDAYÁN ACOSTA', Álvaro TRESIERRA-AYALA', Mildred GARCÍA DÁVILA', Edith RUIZ SÁNCHEZ1, Julia BARDALES GARCÍA', Ricardo REÁTEGUI AMASIFUÉN ${ }^{2}$, Freddy ESPINOZACAMPOS ${ }^{1}$, Carlos DÁVILAFLORES $^{1}$

1 Departamento Académico de Microbiología. Facultad de Ciencias Biológicas. Universidad Nacional de la Amazonía Peruana. Pevas 5ta. Cuadra, Iquitos-Perú. tmoridelaguila@hotmail.com

2 Departamento Académico de Ecología y Conservación. Facultad de Ciencias Forestales. Universidad Nacional de la Amazonía Peruana. Pevas 5ta. Cuadra, Iquitos-Perú.

\section{RESUMEN}

Fue evaluada la población ascomicética y basidiomicética presente en los bosques de la localidad de Puerto Almendras (región Loreto - Amazonía peruana). El estudio fue realizado en una parcela de $800 \mathrm{~m}$ x $200 \mathrm{~m}$, los especímenes fueron colectados mediante el método de los transectos $(4 \times 2)$. La identificación de los 60 especímenes colectados fue realizada mediante claves taxonómicas dicotómicas. Los hongos macroscópicos Basidiomycetes presentaron mayor diversidad de especies (trece especies de Agaricales, nueve especies de Polyporales, una especie de Auriculariales y una especie de Tremellales), que los Ascomycetes (cinco especies del orden Xylariales). En cuanto al tipo de sustrato, la mayor proporción de hongos evaluados en estos bosques fueron de carácter lignícola $(63.3 \%)$, en tanto que los hongos folícolas y terrícolas se presentaron en menor proporción (23.3 \% y 13.4 $\%$, respectivamente).

PALABRAS CLAVE: Basidiomycetes, Ascomycetes, hongos macroscópicos, bosques.

\section{MACROSCOPIC ASCOMYCETES AND BASIDIOMYCETES IN PUERTO ALMENDRAS FORESTS (LORETO, PERÚ).}

\begin{abstract}
Ascomycete and Basidiomycete populations in forests of Puerto Almendras were evaluated in 2011. Within $800 \mathrm{~m} \mathrm{x}$ $200 \mathrm{~m}$ plot, $4 \times 2$ transects, 60 specimens were collected and identified using taxonomic keys. Five species of Ascomycetes, all of them belonging to Xylariales, and twenty four species of Basiodiomycetes (13 species pertaining to Agaricales, 9 Polyporales, 1 Auriculariales and 1 Tremellales, respectively), were identified. Most of them $(63.3 \%)$ were lignicolous, $23.3 \%$ were folicolous and $13.4 \%$ were terricolous.
\end{abstract}

KEYWORDS: Basidiomycetes, Ascomycetes, macroscopic fungus, forests. 


\section{INTRODUCCIÓN}

Los bosques amazónicos se caracterizan por tener una gran diversidad de hábitats, los que brindan las condiciones necesarias para el crecimiento y desarrollo de diversos organismos, entre los que destacan los hongos, haciendo posible la existencia de una gran diversidad de estos organismos eucariotas; pese a ello, estos organismos constituyen un grupo poco estudiado en los ecosistemas amazónicos.

Por lo general, los hongos son benéficos porque contribuyen a la formación del humus, el cual incrementa la fertilidad de los suelos. Por otro lado, pueden servir de complemento en la dieta de las personas, ya que además de la gran cantidad de agua existente en ellos, poseen proteínas, vitaminas, minerales y otras sustancias nutritivas. Algunos son utilizados en medicina tradicional, así como en actividades mágico-religiosas ya que muchas comunidades indígenas lo utilizan en sus rituales (Mata, 2003).

En la naturaleza, los hongos se encuentran sobre distintos substratos: árboles en pie, troncos caídos, césped, hojas caídas y en la tierra, formando grandes poblaciones, atractivas a la vista del ser humano, debido principalmente a sus cuerpos fructíferos muy variados y de diversos colores (Rizzo, 1999).

En los bosques Amazónicos peruanos existe una gran diversidad fúngica, hasta el momento poco estudiado, existiendo reducida información sobre la taxonomía de los hongos macroscópicos (Basidiomycetes y Ascomycetes). En este sentido, el presente trabajo pretende contribuir al conocimiento de estos organismos, generando información sobre la diversidad de los hongos ascomicéticos y basidiomicéticos presente en los bosques de la localidad de Puerto Almendras, ubicada en las proximidades de la ciudad de Iquitos (departamento de Loreto).

\section{MATERIAL Y MÉTODOS}

\section{ÁREA DE ESTUDIO}

Este estudio fue desarrollado en la localidad de Puerto Almendras (distrito de San Juan Bautista, provincia de Maynas, departamento de Loreto). Esta área se encuentra ubicada a la margen derecha del rió Nanay, afluente del rio Amazonas, geográficamente se encuentra entre las coordenadas de $3^{\circ} 49^{\circ} 40^{\prime \prime}$, latitud Sur y $73^{\circ} 22^{\prime} 30^{\prime \prime}$. En dicha área se estableció una parcela de $800 \mathrm{~m} \times 200 \mathrm{~m}$.

\section{PROCEDIMIENTO PARA LA RECOLECCIÓN DE LOS ESPECÍMENES.}

\section{a) Estudio de campo}

Mediante el método de los transectos ( 4 x 2), se colectaron 60 especímenes en la parcela establecida, para lo cual, en una ficha de colecta, se registraron los datos de campo (color de las láminas, presencia de cortinas, restos de velo, escamas, entre otros), ya que con la manipulación o el transporte, podrían modificarse o desaparecer. Se desprendió la seta entera utilizando una espátula y una navaja; y se comprobó si tenía pie, cordones miceliares o volva. En los caracteres organolépticos, se tuvo en cuenta el color de la seta; incluido el de su carne, se observó si producía látex al momento de cortar y si es que cambiaba de color. Se comprobó la textura de la carne, registrando su color inicial y si cambió al cabo de unos minutos.

Se anotó las características del hábitat, tipo de bosque, tipo de substrato en que crecían. Además, se tomó fotografías con la finalidad de no perder sus características originales (Asociación Micológica Joaquim Codina, 2003).

\section{b) Conservación de muestras.}

\section{- Secado.}

Las muestras fueron cubiertas con papel periódico para facilitar el proceso de deshidratación y secadas en una estufa $\left(35-40^{\circ} \mathrm{C}\right)$, por un tiempo aproximado de una semana (según el tamaño y estructura del hongo). Los especímenes pequeños se secaron enteros y los más grandes se cortaron en dos o más partes. Una vez secadas, las muestras se colocaron en el interior de bolsas etiquetadas de papel para protegerlas de las quebraduras, alta humedad, insectos y mohos.

\section{- Conservación en líquido.}

Las muestras que no resistieron la temperatura de secado, se colocaron en un frasco etiquetado de vidrio o de plástico transparente que contenía una solución de formalina: alcohol etílico: ácido acético glacial: agua (75:100:50:75).

\section{c) Procedimientos, técnicas e instrumentos de recolección de datos.}

\section{- Examen macroscópico.}

Se empleó una ficha para anotar las diferentes características observadas y se hizo una descripción de los siguientes datos: Reconocimiento del color de las esporas; la esporada o color de las esporas en masa (Ortega et al., 1996); el tipo de esporóforo; las características del abhimenio o sombrero (medidas, forma, color, margen, tipo de cutícula y si presentaba 
alguna ornamentación); las características del himenio (ornamentaciones, consistencia y color) y las del pie (medidas, forma, la intersección respecto al sombrero, si llevó algún resto de velo, tipo de carne, consistencia, color y si cambió al tocarse) (De Diego, 1979; Asociación Micológica Joaquim Codina, 2003).

\section{-Examen microscópico.}

Para observar las estructuras internas de los hongos se realizaron cortes transversales de las lamelas del himenio, a los que se les añadió una gota de reactivo de Melzer para observar las esporas y $\mathrm{KOH}$ al $10 \%$ para las demás estructuras. Se cubrió con una lámina cubre objeto y se observó en el microscopio binocular y estereoscopio.

Para la observación se tomó en cuenta las diferentes estructuras morfológicas del hongo en estudio, según la Asociación Micológica Joaquim Codina (2003) y Mata et al. (2003), tales como: el basidio, el asca, la basidiospora, la ascospora, el esterigma, los tipos de hifas, etc.

\section{d) Identificación de los especímenes.}

La identificación de los géneros y eventualmente especies colectadas se realizó a través de claves de identificación como: Mata (2003), Keizer (2000), Bécker (1997), Guzmán (1987), Alexopoulos (1985), De Diego (1979), García (1976), Laessoe (2005), Lohmeyer y Künkele (2006), García (2001), Ortega et al. (1996), Pavlich (1976), Pavlich (2001).

\section{e) Clasificación de los hongos macromicetos de acuerdo a la preferencia del sustrato.}

Finalmente, los hongos fueron clasificados de acuerdo a su sustrato: lignícolas, folícolas o terrícolas.

\section{RESULTADOS Y DISCUSIÓN}

Los resultados del presente estudio han posibilitado identificar la existencia de una diversidad fúngica de 29 especies; 5 ascomycetes y 24 basidiomycetes, en los bosques de Puerto Almendras (Tabla 1, Figuras 1 al 3 ). Las 29 especies estaban agrupadas en 17 géneros ( 2 ascomycetes y 15 basidiomycetes) y estos en 14 familias (1 ascomycete y 13 basidiomycetes), incluidas en 5 órdenes (1 ascomycete y 4 basidiomycetes). Esto conlleva a pensar que la población basidiomicética suele ser más diversa. $\mathrm{Al}$ respecto, Espinoza (2004), estudiando la población basidiomicética existente en el área ubicada en el Km. 25 de la carretera Iquitos-Nauta $\left(03^{\circ} 45^{\prime} 01^{\prime \prime}\right.$ L.O. a 126 m.s.n.m.), logró determinar una diversidad mayor ya que reportó 57 especies de Basidiomycetes; sin embargo, cabe mencionar que en el presente trabajo, el número de especies basidiomicética es elevado, con respecto a la población ascomicética. Similar tendencia fue registrada por otros investigadores, pese a que trabajaron en otros ecosistemas, como es el caso de Pavlich (1976), Vasco et al. (2005), Betancurt et al. (2006) y Barrios et al. (2007). Esto conlleva a pensar que los Basidiomycetes constituyen el grupo más complejo entre los hongos, por su inmensa variedad y cantidad (Alexopoulos, 1985).

Se ha determinado que el orden Agaricales es uno de los más numerosos en cuanto a diversidad de especies se refiere, ya que comprende alrededor de 4000 especies. Un aspecto influyente para la existencia de esta alta diversidad y prevalencia encontrada, podría deberse al hecho que sus basidiosporas se eyectan del basidio, los cuales pueden caer al suelo o ser transportados por el viento, favoreciendo su diseminación. La gran variedad de hábitats de los Agaricales, a consecuencia de su amplitud metabólica, posibilita que estos se encuentren, no solamente de modo abundante, sino que suelen mostrar una gran diversidad en muchos bosques (Singer, 1976; Valenzuela et al., 1996; Barría, 2003), lo cual ha sido corroborado en el presente estudio.

En lo que concierne a las características ecológicas de los Ascomycetes y Basidiomycetes encontrados en la totalidad del área estudiada, la mayoría de ellos (63.3 $\%$ ), fueron registrados como lignícolas (hongos que crecen sobre árboles en descomposición), el $23.3 \%$ se clasificó como folícolas y la menor parte de la población $(13.3 \%$ ), fue considerada como terrícola. Estos resultados guardan relación con los reportados por Espinoza (2004) y Channona et al. (2007), quienes indicaron que el tipo de substrato más utilizado por los macromicetos son los árboles en descomposición, reportando la presencia de hongos lignícolas en porcentajes de $56 \%$ y $57,67 \%$, respectivamente. 
Tabla 1. Diversidad ascomicética y basidiomicética encontrada en los bosques de la localidad de Puerto Almendras (departamento de Loreto, Amazonía peruana).

\begin{tabular}{|c|c|c|c|c|}
\hline DIVISIÓN & ORDEN & FAMILIA & GÉNERO & ESPECIE \\
\hline \multirow[b]{2}{*}{ Ascomycota } & \multirow[b]{2}{*}{ Xylariales } & \multirow[b]{2}{*}{ Xylariaceae } & Camillea & C. leprieurii \\
\hline & & & Xylaria & $\begin{array}{l}\text { X. polymorpha } \\
X . \text { hypoxylon } \\
\text { X. fuckei } \\
\text { X. cubensis }\end{array}$ \\
\hline \multirow{15}{*}{ Basidiomycota } & \multirow{6}{*}{ Polyporales } & \multirow[t]{2}{*}{ Ganodermataceae } & Amauroderma & $\begin{array}{l}\text { A. ocellatum } \\
\text { A. schomburgkii }\end{array}$ \\
\hline & & & Ganoderma & G. applanatum \\
\hline & & \multirow{2}{*}{ Coriolaceae } & Hydnopolyporus & H. palmatus \\
\hline & & & Trametes & T. elegans \\
\hline & & Polyporaceae & Polyporus & $\begin{array}{l}\text { P. schweinitzii } \\
\text { P. sanguineus } \\
\text { P. tenuiculus }\end{array}$ \\
\hline & & Lentinaceae & Lentinus & L. crinitus \\
\hline & Auriculariales & Auriculariaceae & Auricularia & A. delicada \\
\hline & \multirow{7}{*}{ Agaricales } & Psathyrellaceae & Coprinus & $\begin{array}{l}\text { C. disseminatus } \\
\text { C. niveus }\end{array}$ \\
\hline & & Hygrophoraceae & Hygrocybe & H. virginea \\
\hline & & Marasmiaceae & Marasmius & $\begin{array}{l}\text { M. foetidum } \\
\text { M. rotula } \\
\text { M. corrugata } \\
\text { M. cladophyllus } \\
\text { M. bertori } \\
\text { M. candidus }\end{array}$ \\
\hline & & Tricholomataceae & Mycena & M. rosella \\
\hline & & Physalacriaceae & Oudemansiella & 0. canarii \\
\hline & & Pleurotaceae & Pleurotus & P. ostreatus \\
\hline & & Psathyrellaceae & Psathyrella & P. candolleana \\
\hline & Tremellales & Tremellaceae & Tremella & T. mesenterica \\
\hline
\end{tabular}



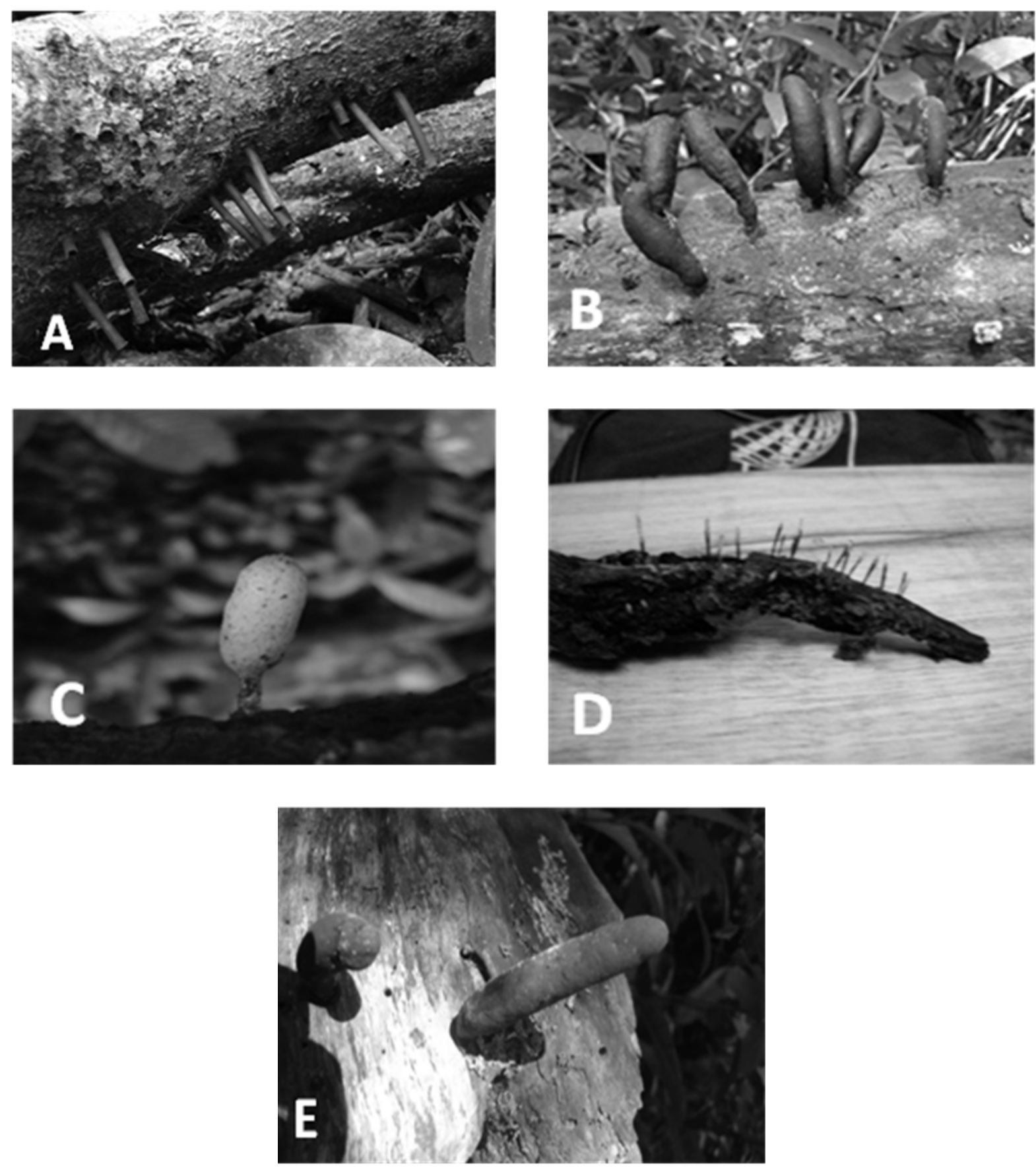

Figura 1. Especies de Ascomycetes encontrados en los bosques de Puerto Almendras (Departamento de Loreto, Amazonía peruana): $\mathrm{A}=$ Camillea lepriuri; $\mathrm{B}=$ Xylaria polymorpha; $\mathrm{C}=$ Xylaria fuckei; $\mathrm{D}=$ Xylaria hypoxylon; $\mathrm{E}=$ Xylaria cubensis. 
FOLIA

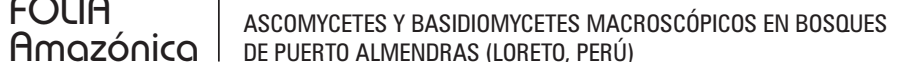
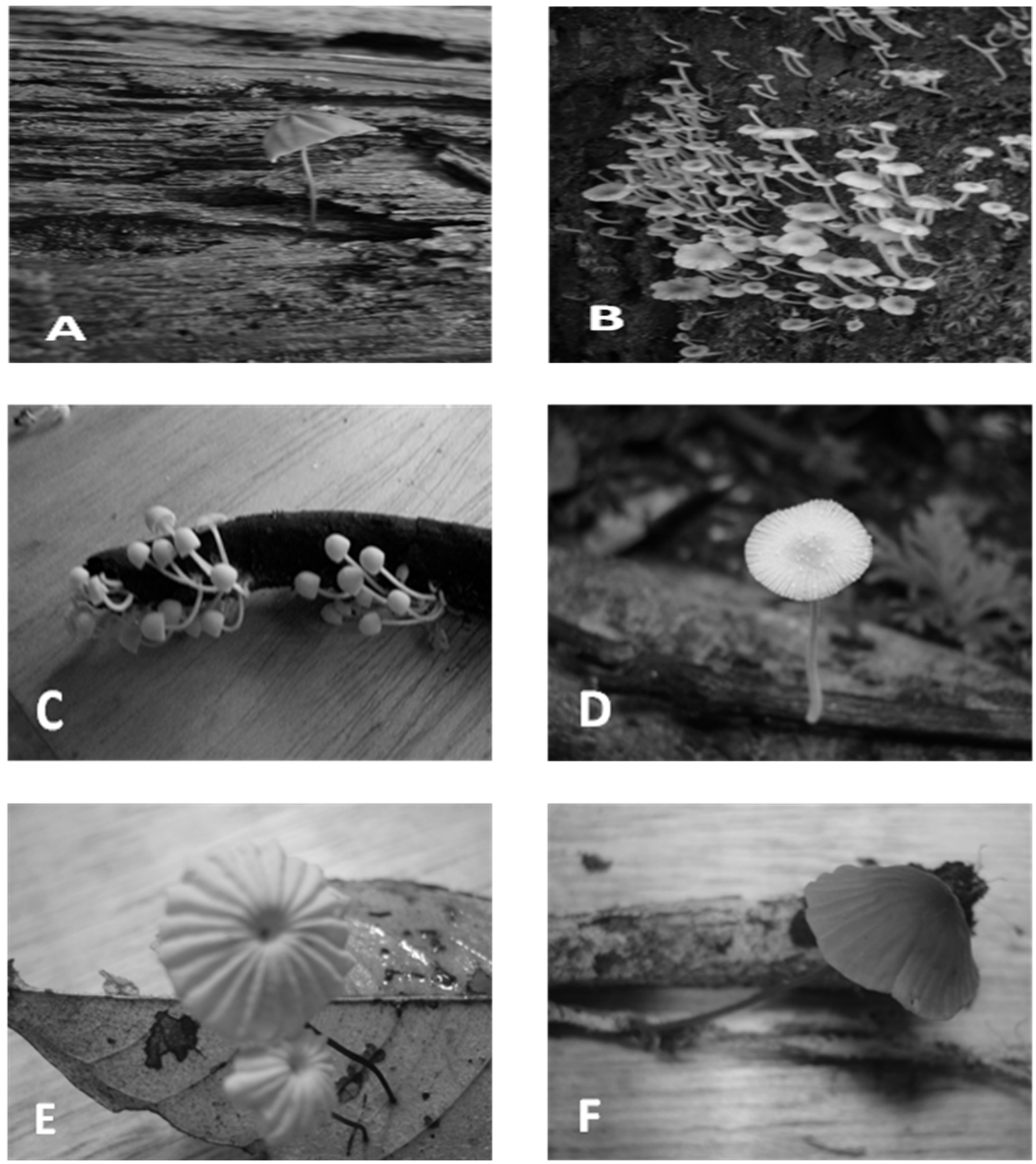

Figura 2. Especies de Basidiomycetes encontrados en los bosques de Puerto Almendras (Departamento de Loreto, Amazonía peruana): $\mathrm{A}=$ Marasmius bertori; $\mathrm{B}=$ Hydrocybe virginea; $\mathrm{C}=$ Coprinus disseminatus; $\mathrm{D}=$ Coprinus niveus $; \mathrm{E}=$ Marasmius rotula; $\mathrm{F}=$ Mycena rosella. 

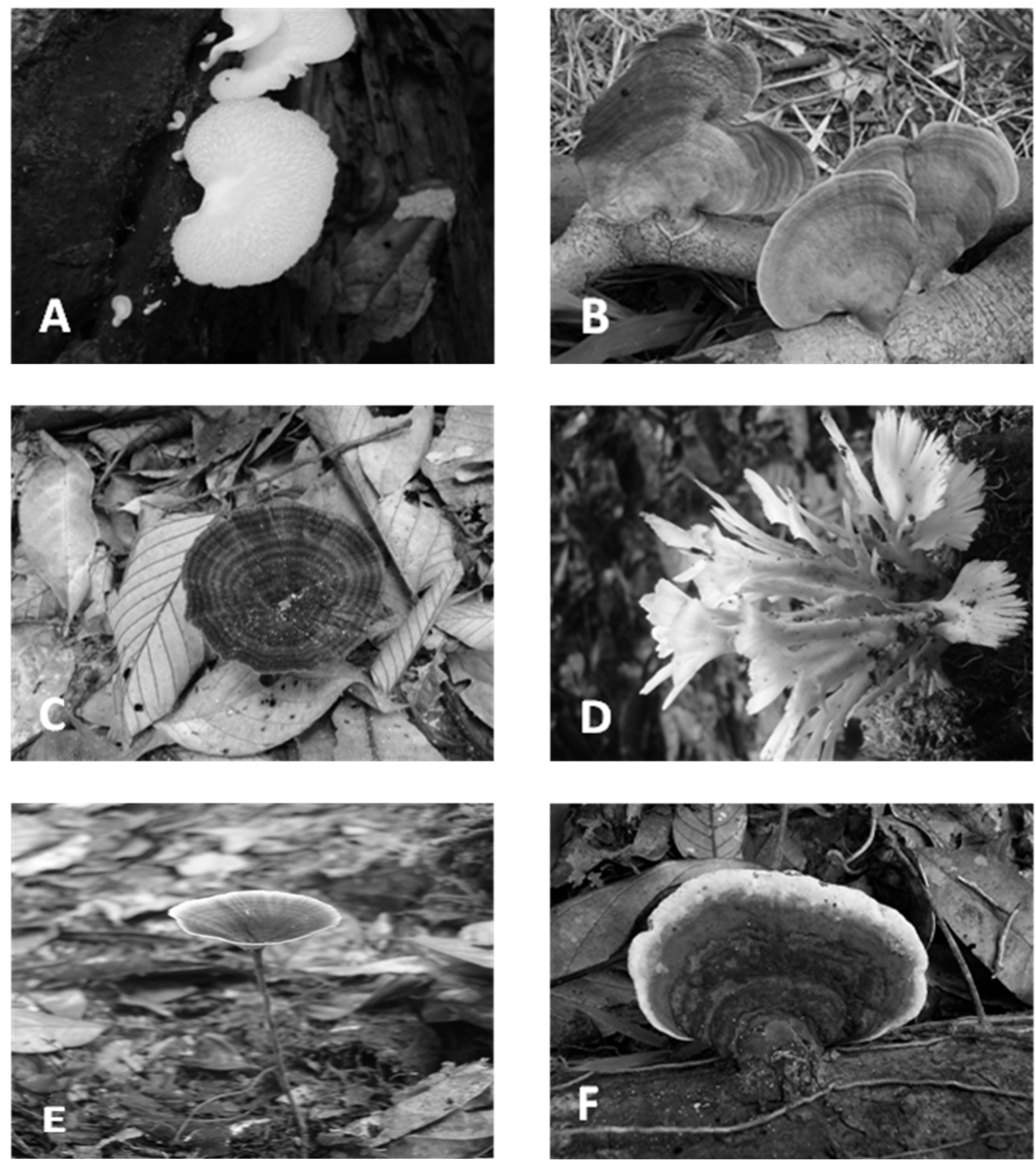

Figura 3. Especies de Basidiomycetes encontrados en los bosques de Puerto Almendras (Departamento de Loreto, Amazonía peruana): $\mathrm{A}=$ Polyporus tenuiculus; $\mathrm{B}=$ Polyporus sanguineus; $\mathrm{C}=$ Polyporus schweinitzii; $\mathrm{D}$ $=$ Hydnopolyporus palmatus; $\mathrm{E}=$ Amauroderma ocellatum; $\mathrm{F}=$ Ganoderma applanatum. 


\section{BIBLIOGRAFÍA CITADA}

Alexopoulos, C. 1985. Introducción a la Micología. Ed. Omega S.A. España. 638pp.

Asociación Micológica Joaquím Codina. 2003. Iniciación a la Micología Universitaria de Girona. Facultad de Ciencias-Laboratorio de Botánica (PB7) Campus de Moltilivi.17001, Girona. 20pp.

Barría, D.F. 2003. Diversidad y abundancia de Agaricales $S$. $L$. en parcelas fertilizadas con $\mathrm{NH}_{4} \mathrm{NO}_{3}$ en un bosque de Nothofagus obliqua (Mirb) Oerst. de la $\mathrm{X}^{\mathrm{a}}$ Región, Chile. (http://cybertesis.uach.cl/tesis/uach/2003/fcb275 d.pdf). Acceso: 12/03/2012.

Barrios, R.; Quezada, M.; López, R.; Fuentes, A. 2007. Fortalecimiento en el conocimiento taxonómico de macrohongos tropicales de Guatemala. (http://digi.usac.edu.gt/bvirtual/ investigacio files/INFORMES/PUIRNA/INF2007-011.pdf). Acceso: 03/05/2012.

Bécker, G. 1997. Hongos y Setas de Europa. Susaeta Ediciones, S.A. España. 319pp.

Betancurt, M.; Calderón, M.; Betancurt, O.; Suerquia, A. 2006. Hongos macromycetes en dos relictos de bosque húmedo tropical montano bajo de la vereda la Cuchilla, Marmato, Caldas. Colombia. (). Acceso: 22/05/2012.

Chanona, F; Andrade, R; Castellanos, J.; Sánchez, J. 2007. Macromicetos del Parque Educativo Laguna Bélgica, municipio de Ocozocoautla de Espinoza, Chiapas, México. Revista Mexicana de Biodiversidad, 78: 369-381.

De Diego, F. 1979. Setas (Hongos). Ediciones MundiPrensa. España. 309pp.

Keizer, J. 2000. La enciclopedia de las setas. Madrid España, 286pp.

Espinoza, M. 2004. Determinación de Hongos de la Clase Basidiomycetes en el Centro de Investigación Allpahuayo Loreto - Perú. Tesis de pre-grado. Universidad Nacional de la Amazonía Peruana. Iquitos, Perú. $125 \mathrm{pp}$.

García, M. 1976. Hongos de la madera (basidiomicetos). Primera edición. Publicaciones de extensión agraria. Ajenjos S.A. Madrid España. 243pp.
García, M. 2001. Manual para buscar setas. Ministerio de Agricultura, Pesca y Alimentación. Madrid España. 454pp.

Guzmán, G. 1987. Identificación de los hongos comestibles, venenosos alucinantes y destructores de la madera. Cuarta reimpresión. Edit LIMUSA. México. 451pp.

Laessoe, T. 2005. Hongos: Manuales de identificación. Ediciones Omega SA, Barcelona. España. 304pp.

Lohmeyer, T.; Künkele, U. 2006. Setas. Barcelona. España. 255pp.

Mata, M. 2003. Macrohongos de Costa Rica. Instituto Nacional de Biodiversidad. INBio, Edit. INBio, Santo Domingo de Heredia, Costa Rica. Vol 1. 255pp.

Ortega, A.; Piqueras, J.; Amate, P. 1996. Setas: Identificación, Toxicidad, Gastromicología. Proyecto Sur de Ediciones. España. 475pp.

Pavlich, M. 1976. Ascomycetes y Basidiomycetes del Perú. Con énfasis de especies de la ceja de montaña y selva tropical. Memorias del Museo de Historia Natural "Javier Prado" No 17. UNMSM. LimaPerú. 89pp.

Pavlich, M. 2001. Los hongos comestibles del Perú. BIOTA, 100:3-19.

Rizzo, E. 1999. Estudio taxonómico de la mycobiota del parque arqueológico Tikal. Tesis de pre-grado. Universidad de San Carlos de Guatemala. 74pp.

Singer, R. 1976. Flora Neotropical. The New York Botanical Gardens, New York. 347pp.

Valenzuela, E.; Ramírez, C.; Moreno, G.; Polette, M.; Garnica, S.; Peredo, H.; Grinbergs, J. 1996. Agaricales más comunes recolectados en el Campus Isla Teja de la Universidad Austral de Chile. Bosque, 17: 51-63.

Vasco, A.; Franco, A.; López, C.; Boekhout, T. 2005. Macromycetes (Ascomycota, Basidiomycota) de la región del medio Caquetá departamentos de Caquetá y Amazonas (Colombia).

(http://www.siac.net.co/biota/bitstream/123456789/1

53/1/HONGOS_2212205.pdf). Acceso:22/04/2012. 